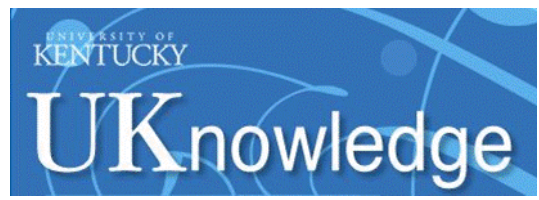

University of Kentucky

UKnowledge

Institute for Biomedical Informatics Faculty

Publications

Institute for Biomedical Informatics

2-17-2017

\title{
HyCLASSS: A Hybrid Classifier for Automatic Sleep Stage Scoring
}

\author{
Xiaojin Li \\ University of Kentucky, xiaojin.li@uky.edu \\ Licong Cui \\ University of Kentucky, licong.cui@uky.edu \\ Shiqiang Tao \\ University of Kentucky, shiqiang.tao@uky.edu \\ Jing Chen \\ Lanzhou University, China \\ Xiang Zhang \\ Pennsylvania State University
}

See next page for additional authors

Follow this and additional works at: https://uknowledge.uky.edu/bmi_facpub

Part of the Bioinformatics Commons

Right click to open a feedback form in a new tab to let us know how this document benefits you.

\section{Repository Citation}

Li, Xiaojin; Cui, Licong; Tao, Shiqiang; Chen, Jing; Zhang, Xiang; and Zhang, Guo-Qiang, "HyCLASSS: A Hybrid Classifier for Automatic Sleep Stage Scoring" (2017). Institute for Biomedical Informatics Faculty Publications. 6.

https://uknowledge.uky.edu/bmi_facpub/6

This Article is brought to you for free and open access by the Institute for Biomedical Informatics at UKnowledge. It has been accepted for inclusion in Institute for Biomedical Informatics Faculty Publications by an authorized administrator of UKnowledge. For more information, please contact UKnowledge@lsv.uky.edu. 


\section{HyCLASSS: A Hybrid Classifier for Automatic Sleep Stage Scoring}

Digital Object Identifier (DOI)

https://doi.org/10.1109/JBHI.2017.2668993

\section{Notes/Citation Information}

Published in IEEE Journal of Biomedical and Health Informatics, v. 22, no. 2, p. 375-385.

(C) 2017 IEEE

The copyright holder has granted the permission for posting the article here.

Authors

Xiaojin Li, Licong Cui, Shiqiang Tao, Jing Chen, Xiang Zhang, and Guo-Qiang Zhang 


\title{
HyCLASSS: A Hybrid Classifier for Automatic Sleep Stage Scoring
}

\author{
Xiaojin Li ${ }^{\circledR}$, Licong Cui, Shiqiang Tao, Jing Chen, Xiang Zhang, and Guo-Qiang Zhang ${ }^{\mathbb{1}}$
}

\begin{abstract}
Automatic identification of sleep stage is an important step in a sleep study. In this paper, we propose a hybrid automatic sleep stage scoring approach, named HyCLASSS, based on single channel electroencephalogram (EEG). HyCLASSS, for the first time, leverages both signal and stage transition features of human sleep for automatic identification of sleep stages. HyCLASSS consists of two parts: A random forest classifier and correction rules. Random forest classifier is trained using $30 \mathrm{EEG}$ signal features, including temporal, frequency, and nonlinear features. The correction rules are constructed based on stage transition feature, importing the continuity property of sleep, and characteristic of sleep stage transition. Compared with the gold standard of manual scoring using Rechtschaffen and Kales criterion, the overall accuracy and kappa coefficient applied on 198 subjects has reached $85.95 \%$ and 0.8046 in our experiment, respectively. The performance of HyCLASS compared favorably to previous work, and it could be integrated with sleep evaluation or sleep diagnosis system in the future.
\end{abstract}

Index Terms-Automatic sleep stage scoring, EEG, hybrid classifier, PSG, sleep stage transition.

\section{INTRODUCTION}

$\mathbf{S}$ LEEP is one of the most important circadian rhythms of human physiological activities [1]. It has a sequence of sleep stages related to autonomous nervous system functions [2]. The quality and quantity of sleep impacts the performance of many basic activities, such as learning, memorization, and

Manuscript received July 8, 2016; revised December 10, 2016 and January 31, 2017; accepted February 6, 2017. Date of publication February 17, 2017; date of current version March 5, 2018. This work was supported in part by the University of Kentucky, in part by the Center for Clinical and Translational Science Award UL1TR001998, in part by the National Sleep Research Resource under Grant NHLBI R24 HL114473, and in part by the National Science Foundation under Grant 1626364. (Corresponding author: Guo-Qiang Zhang.)

$\mathrm{X}$. $\mathrm{Li}$ is with the Department of Electrical Engineering and Computer Science, Case Western Reserve University, Cleveland, OH 44106-7078 USA, and also with the Institute for Biomedical Informatics, University of Kentucky, Lexington, KY 40506 USA (e-mail: xx1509@ case.edu).

L. Cui is with the Department of Computer Science, University of Kentucky, Lexington, KY 40506 USA (e-mail: licong.cui@uky.edu).

S. Tao and G.-Q. Zhang are with the Institute for Biomedical Informatics, University of Kentucky, Lexington, KY 40506 USA (e-mail: strongtsq@gmail.com; gqatcase@gmail.com).

$\mathrm{J}$. Chen is with the School of Information Science and Engineering, Lanzhou University, Lanzhou 730000, China (e-mail: chen.j15@uky. edu).

$X$. Zhang is with the College of Information Sciences and Technology, Pennsylvania State University, University Park, PA 16801 USA (e-mail: xzhang@ist.psu.edu).

Digital Object Identifier 10.1109/JBHI.2017.2668993 concentration [3]. Better understanding of some sleep disorders, such as insomnia and sleep apnea, relies on accurate detection of sleep stages and the sleep cycle. In sleep studies for clinical diagnosis and treatment of sleep disturbances, the first step is identification of an individual's sleep stages [4].

Traditionally, domain experts visually analyze and manually score the recorded over-night polysomnography (PSG) signals, such as electroencephalogram (EEG), electrocardiogram (ECG), electromyography (EMG), electrooculogram (EOG), pulse oximetry, and respiration. Normal practice divides the entire sleep record into 30-second epochs and each epoch is assigned a certain sleep stage according to some standard criterions, such as Rechtschaffen and Kales (R\&K) rules [5]. Such manual scoring method remains the standard practice [6]. Based on R\&K rules, sleep stages can be categorized by wakefulness (Wake), rapid eye movement (REM), and nonrapid eye movement (NREM). NREM can be categorized into stages 1, 2, 3 and 4 . Stages 3 and 4 are often combined together and referred to deep sleep, or slow wave sleep (SWS) [7].

However, manual stage scoring on PSG by domain experts is time-consuming and labor-intensive. Each hour of sleep requires the scoring of 120 epochs. The National Sleep Research Resource (NSRR) [8], [9], which is a large-scale integrated sleep data repository, consists of a collection of sleep research data across the United States, with thousands of hours of sleeprelated data. Thus, developing a simple and reliable automatic sleep stage scoring system can reduce significant manual work in sleep studies. Several automatic sleep stage scoring approaches have been proposed, including

1) time domain analysis [10], [11];

2) frequency domain analysis [4], [7], [12]-[14];

3) time-frequency analysis including wavelet transform [15]-[18];

4) EEG non-linear analysis [7], [19], [20];

5) decision tree (DT) [14], [21];

6) artificial neural network (ANN) [12], [18];

7) fuzzy clustering [4];

8) k-nearest neighbor (KNN) [16], [22];

9) support vector machine (SVM) [7];

10) random forest [15];

11) hidden markov model (HMM) [23]-[25];

12) deep belief network (DBN) [13].

These methods reported accuracies ranging from $67 \%$ to $86 \%$, compared with experts' score. However, most of the existing work involved a small number of sleep records in dataset (less 
than 30 subjects), which may cause bias and low generalizability. Moreover, the existing work only focused on analyzing signal features without considering the dynamic stage transition information. Jääskinen et al. [6] performed a study on the distribution of transitions from one stage to another using Markov model, and they discovered a distribution difference between normal and recovery sleep. Additionally, stage dynamic transition information reveals significant characteristics of the degree of sleep continuity [26] and potentially provides valuable insights for better understanding of the mechanisms of sleep stage interactions [27]. The goal of this paper is to formulate and test this main hypothesis at a large scale: the properties of dynamic stage transition could improve the performance of sleep stage scoring.

We propose a hybrid classifier HyCLASSS, for the first time, leverages both signal and stage transition features of human sleep for automatic identification of sleep stages. HyCLASSS consists of two parts: random forest classifier and correction rules. We first train a random forest classifier using three types of features from EEG signal: time-domain features, frequencydomain features, and non-linear features. We then construct and apply the correction rules based on a proposed stage transition graph representing the dynamic characteristic of sleep stage transition. Combining both signal and stage transition features, HyCLASSS can overcome the limitations of the previous methods and improve the performance. In this study, we divided sleep stages into five categories: wakefulness (Wake), rapid eye movement (REM), sleep stage 1 (S1), sleep stage 2 (S2), and slow wave sleep (SWS). The overall accuracy, kappa coefficient [28], [29], precision, recall and F1-score [30], [31] are calculated to demonstrate the performance of HyCLASSS. The overall accuracy and kappa coefficient of our approach applied to 198 subjects (from two existing cohort studies) are $85.95 \%$ and 0.8046 , respectively, which compared favorable with pervious studies.

The rest of this paper is organized as follows. Section II describes the background of automatic sleep stage scoring. Section III describes the materials and methods. Section IV discusses the results. Section V provides a brief comparison with other related work and discusses future work.

\section{BACKGROUND}

Different types of approach exist for time series classification, such as raw data-based, model-based, and feature-based [32]. For automatic sleep stage scoring, feature-based method has been the most popular, involving two major steps: feature extraction and classifier construction [33].

\section{A. Feature Extraction From EEG Signal}

For EEG signal feature extraction, most of the reported features in previous studies are considered in this study. These features can be grouped into three categories: 1) time-domain features, 2) frequency-domain features, and 3) non-linear features.

1) Time-Domain Features: Time-domain features include statistical measures and Hjorth parameters [34]. Statistical measures include mean, kurtosis, and skewness evaluating statistical characteristics [35]. The mean statistic measures the central tendency of a probability distribution. Kurtosis feature measures the tailedness of a probability distribution and describes the shape of a probability distribution. Skewness measures the asymmetry of a probability distribution. Hjorth parameters are generally used in feature extraction for EEG signal analysis [36] including activity, mobility, and complexity [37]. The activity measures the variance of a time function. The mobility infers to the mean frequency or the proportion of standard deviation of the power spectrum. The complexity measures the change in frequency.

2) Frequency-Domain Features: EEG signals of each sleep stage have various behaviors in different frequency bands, and the characteristics of different sleep stages in different frequency bands are reported in many previous work (see [33] for a survey). Previous studies demonstrated that spectral power is an important feature for automatic sleep stage scoring [15]. For instance, the power of lower frequencies of EEG becomes stronger with the increasing depth of sleep [38], the EEG signal of stage 1 has higher amplitude between 2-7 Hz. Stage 2 can be characterized by the presence of sleep spindles band (12-14 Hz). SWS is scored when there is low frequency (less than $2 \mathrm{~Hz}$ ) waves.

Additionally, the average signal amplitudes in specific frequency bands by applying band pass filter, for instance, the $0.5-1.0 \mathrm{~Hz}$ band, was recorded as a feature.

3) Non-Linear Features: All the features mentioned above are linear features. Non-linear and dynamical properties are also important in representing the behaviors of EEG signal [19], such as Shannon entropy and sample entropy. Various researchers in the past [20], [39], [40] demonstrated their relevance for sleep stage scoring and EEG characterization.

\section{B. Random Forest Classifier}

Random forest classifier is an ensemble learning method for classification by constructing a group of decision trees. Its output is the mode of the classes of the individual trees, and it has been proposed for automatic sleep stage scoring [15]. The overall output is determined by applying the object to each tree and selecting the classification with the most weighted votes. The weight of each tree is adjusted using misclassification and outof-bag measures.

A random forest classifier has three main properties [41]: adaptability, scalability, and robustness. Other classifiers typically do not have all the three properties (see Table I).

Adaptability: A random forest classifier estimates the importance of variables and provides a way for tuning with additional training data by assigning different weights for each decision tree. In comparison, ANN is not as adaptable, since its parameters are sensitive to the data, and it is difficult for ANN to be tuned to new datasets for constructing a well-learned neural network [42].

Scalability: A random forest classifier can handle thousands of input variables and work efficiently on large datasets. In contrast, SVM has poor performance on large datasets since the 
TABLE I

PROPERTIES OF CLASSIFIERS

\begin{tabular}{lccc}
\hline \hline & Adaptability & Scalability & Robustness \\
\hline KNN & Yes & No & No \\
Decision tree & Yes & No & No \\
ANN & No & No & Yes \\
SVM & Yes & No & Yes \\
Random forest & Yes & Yes & Yes \\
\hline \hline
\end{tabular}

speed of convergence can be low [43]. Similarly, KNN and ANN have limited scalability and are not suitable for large datasets due to work involved in training and testing. Although training and testing for a single decision tree can be efficient, it comes with the drawback of overfitting.

Robustness: A random forest classifier can balance error in datasets with unbalanced class population. A single decision tree classifier is sensitive to noise and outliers in the data as a slight change in training data can result in a different tree. Therefore, single decision tree's performance with unbalanced training data is not optimal [44]. KNN also cannot handle unbalanced training dataset well since the classification result depends on the number and the class label of nearest neighbors. KNN typically cannot perform optimally for groups with less training data.

\section{Sleep Stage Transition}

Dynamic sleep stage transition describes the relationship between one sleep stage and another. Most existing sleep stage classification studies only used polysomnography signals, such as EEG and EMG, for feature extraction and ignored the sleep stage transition features. In [26], the study proved that sleep stage transition, beyond conventional PSG measures of sleep, could be used to characterize sleep disruption. It also indicated that sleep stage transition rates provided additional valuable information of sleep continuity, and it was useful in predicting specific outcomes. Stage transition provides additional useful information for classification and prediction; however, one of the challenges is that it is difficult to learn from dataset.

In [14], the authors applied a smoothing process on classified results to improve their classifier's performance, which considers the continuity of a subject's sleep. However, the smoothing process only consisted of several rules manually extracted from dataset. In this paper, HyCLASSS solves this problem through extracting sleep stage transition features from sleep stage sequences automatically with a Markov model.

\section{Materials And Methods}

HyCLASSS consists of several steps, and its workflow is shown in Fig. 1. Pre-processing the EEG signal starts the process (Step 1), followed by feature extraction (Step 2). After feature extraction, a hybrid classifier is trained and tested using extracted features (Step 3-4). All the steps are implemented using Matlab (Mathworks, Inc.-USA).

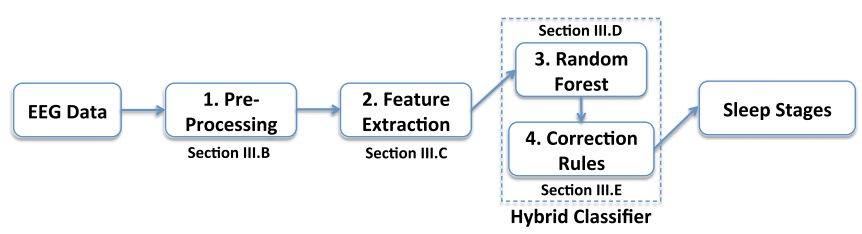

Fig. 1. HyCLASSS workflow.

\section{A. Subjects}

This study uses a dataset consists of subjects: 1) 116 subjects (57 males and 59 females) selected from the Cleveland Children's Sleep and Health Study (CCSHS) [45], which was focused on teenagers with an age range of 16-19 years old, and 2) 82 subjects ( 35 males and 47 females) selected from the Cleveland Family Study (CFS) [46], which was focused on subjects with an age range of 6-88 years old. Actually, 84 subjects were selected from CFS (totally 200 subjects); however, two of them were removed since they have data quality issues due to unlabeled parts of their signal. Both datasets are available at National Sleep Research Resource.

The signal recordings were done for an entire night of sleep (8$12 \mathrm{~h}$ ). The EEG channel was recorded from the C4-A2 channel sampled at $128 \mathrm{~Hz}$. The sleep stages were manually scored in epochs of 30s according to the Rechtschaffen and Kales (R\&K) standard. The sleep stages are divided into five categories: Wake, REM, S1, S2, and SWS.

\section{B. Pre-Processing}

Muscle artifact, eye movements and eye blinking are the common physiological artifacts present in the EEG recordings, and these artifacts make interpretations of the EEG signal difficult [7]. In order to minimize residual artifacts presence, the signals are filtered with a band pass filter with cutoff frequencies at $0.2 \mathrm{~Hz}$ and at $35 \mathrm{~Hz}$.

\section{Feature Extraction}

We extracted 33 features including time-domain, frequencydomain and non-linear features: 1) six time-domain features (mean, kurtosis, skewness, activity, mobility, and complexity), 2) spectral power and spectral amplitude of 11 frequency bands (total 22 features), and 3) five non-linear features. Then we removed three features: mean, mobility and complexity, since they had similar values for each stage and were not useful for classification; finally, 30 features were used in HyCLASSS.

The spectral power in several significant frequency bands is calculated by fast Fourier transforms (FFT) for each epoch, and the total spectral power for each band is used as a frequency feature. All frequency bands with their extraction details are described in Table II. To represent non-linear characteristics of EEG, five non-linear dynamics features are extracted, namely, C0 Complexity [47], [48], Shannon Entropy [49], [50], the largest Lyapunov exponent [51], Spectral Entropy [52], [53], and Kolmogorov Entropy [54]. 
TABLE II

EXTRACTED EEG FREQUENCY BANDS

\begin{tabular}{lccccc}
\hline \hline Name & Notation & $\mathrm{Hz}$ & Name & Notation & $\mathrm{Hz}$ \\
\hline Slow- & & & Spindle & A_spindle & {$[10.5,14.5]$} \\
Oscillations & A_slwoc & {$[0.5,2.0]$} & Sigma & A_sigma & {$[12.0,15.0]$} \\
SWA & A_SWA & {$[0.5,5.5]$} & Slow Sigma & A_slwsig & {$[12.0,13.5]$} \\
Delta & A_delta & {$[1.0,4.0]$} & Fast Sigma & A_fstsig & {$[13.5,15.0]$} \\
Theta & A_theta & {$[4.0,8.0]$} & Beta1 & A_betal & {$[15.0,20.0]$} \\
Alpha & A_alpha & {$[8.0,10.5]$} & Beta2 & A_beta2 & {$[20.0,30.0]$} \\
\hline \hline
\end{tabular}

\begin{tabular}{|c|c|c|c|c|c|c|c|c|c|c|}
\hline Feature values & 0.7 & 0.3 & 0.9 & 0.5 & 0.1 & 0.4 & 0.6 & 0.8 & 0.2 & 1.0 \\
\hline \multicolumn{11}{|c|}{ Recording Index } \\
\hline Index & 1 & 2 & 3 & 4 & 5 & 6 & 7 & 8 & 9 & 10 \\
\hline Feature values & 0.7 & 0.3 & 0.9 & 0.5 & 0.1 & 0.4 & 0.6 & 0.8 & 0.2 & 1.0 \\
\hline \multicolumn{11}{|c|}{ Sorting according to feature values } \\
\hline Index & 5 & 9 & 2 & 6 & 4 & 7 & 1 & 8 & 3 & 10 \\
\hline Feature values & 0.1 & 0.2 & 0.3 & 0.4 & 0.5 & 0.6 & 0.7 & 0.8 & 0.9 & 1.0 \\
\hline \multicolumn{11}{|c|}{ Apply 5-bin } \\
\hline Index & 5 & 9 & 2 & 6 & 4 & 7 & 1 & 8 & 3 & 10 \\
\hline Feature values & 1 & 1 & 2 & 2 & 3 & 3 & 4 & 4 & 5 & 5 \\
\hline \multicolumn{11}{|c|}{ Obtain the final ranked feature } \\
\hline Index & 1 & 2 & 3 & 4 & 5 & 6 & 7 & 8 & 9 & 10 \\
\hline Feature values & 4 & 2 & 5 & 3 & 1 & 2 & 3 & 4 & 1 & 5 \\
\hline
\end{tabular}

Fig. 2. An illustrative example of $\mathrm{N}$-bin procedure $(\mathrm{N}=5)$.

After feature extraction, unit norm is applied to normalize features to $0-1$ ranges to reduce the effects of individual variability. Additionally, for a subject, each feature vector of all epochs is ranked by N-bin approach to reduce the complexity and running time of building trees in random forest. The procedure for N-bin approach is summarized in the following steps: 1) record the index of original feature vector, and then sort the feature vector, 2) divide the feature vector into $\mathrm{N}$ bins of the same size, 3) the elements in the same bin have the same rank level, 4) using recorded index to obtain the final ranked feature. Fig. 2 shows an example of this procedure of 5-bin. The selection of $\mathrm{N}$ is described in Section IV-B.

\section{Random Forest}

As mentioned in Section II-B, a random forest classifier has many advantages, such as adaptable, scalability and robustness. We experimented with several machine learning classification, including KNN, C4.5 decision tree and random forest, found the random forest technique provided the best performance, therefore, we chose it for HyCLASSS.

We used four processes to build the random forest [41]. First, build each decision tree using method, such as classification and regression tree, random trees, and C4.5.

Second, in the construction of each decision tree, nodes and leaves are built by selecting a random number of features. This process will minimize the correlation among the features and decrease the sensitivity to noise; in this way, the accuracy of classification will be increased [15].
Third, apply the technique of bootstrap (sampling with replacement) to sample the sub-training set for each tree from the whole training data set. In other words, each tree is constructed using bootstrap sample technique, and the variance of the model can be reduced without increasing the bias. It is easy to obtain strongly correlated trees by training all trees with the same training set, and bootstrap sampling is a good way to de-correlate the trees. For a single decision tree, its prediction results may be highly sensitive to the noise in its particular training set; however, in random forest, the average of all trees is less sensitive to noise since the trees are more de-correlated. Additionally, during the training process of each tree, about two-third of the sub-training set is used in the construction of the tree; the remaining one-third part is used to test the classification performance of the tree, and this is called "the out-of-bag error estimate." Therefore, it gets an unbiased estimate of the test set error internally in random forest, and there is no need to use further cross-validation [41].

Fourth, predict the classification for a new object $O$ by weighting the predictions from all the individual trees on $O$.

In this study, we used a random forest with 400 trees in our classifier. Parameter selection is discussed in Section IV-B, which shows that classification results varied little with respect to parameters.

\section{E. Construction of Correction Rules}

As mentioned in Section II-C, sleep stage transition can provide additional information for classification. Intuitively, changing of sleep stages during night sleep should not be random, and some transitions may not happen. For example, [REM, SWS, S2] rarely occurs in sleep stage transition. To verify this intuition, we designed a simple experiment to compare the sleep stage sequence with a random sequence using Kolmogorov complexity [55]-[57], which is a popular measurement to evaluate the randomness of sequence. The results indicate that the stage sequence of whole night sleep is not a random sequence. Section V describes the details.

[14] applied a smoothing process on classified results to improve their classifier's performance. It considered the temporal contextual information and improved the continuity of a subject's sleep stage scoring results. Some correction rules were constructed according to the relationship among current epoch, prior-epoch and posterior-epoch [14]. For example, three consecutive epochs of [S1, REM, S2] were replaced with the sequence [S1, S1, S2]. Similarly, consecutive epochs of [S1, S2, S1] were replaced with the sequence [S1, S1, S1] [14], [58]. However, their correction rules only contain a few manually defined rules and need more prior knowledge of the sleep study and characteristics of the sleep dataset.

This begs the question: can we automatically build the general correction rules based on the data itself with less prior knowledge? Therefore, we provide a novel method to automatically construct correction rules that only depend on the dataset using a Markov model. Then, apply those rules on the output of the random forest classifier. The Markov model was used to find out the transition distribution from one stage to another [6] and 


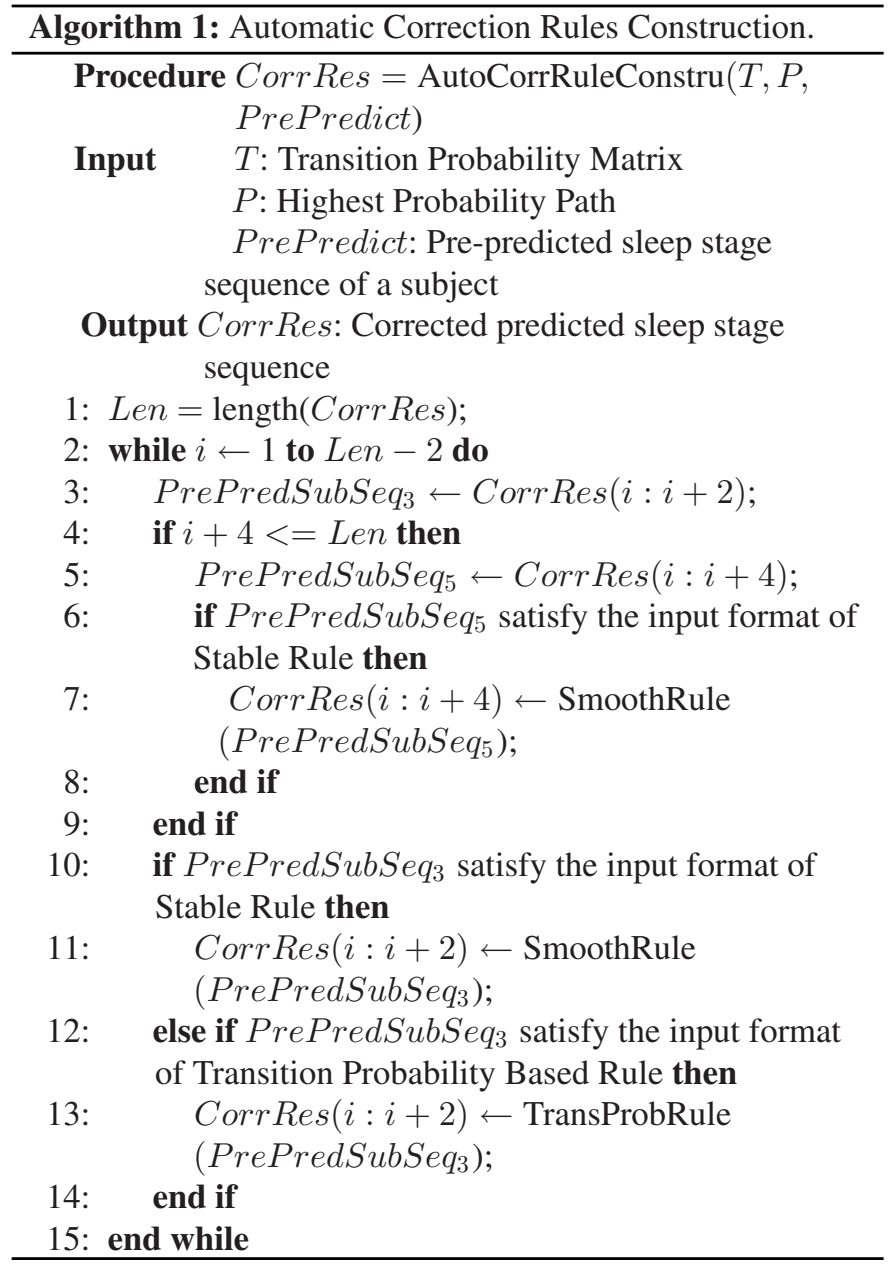

provided a compact and interpretable representation of the sleep stages in a dynamic sleep process. The results are described in Section IV.

Our correction rules can be divided into two parts, namely, Smoothing rule and Transition-probability-based rule. Each rule represents different characteristics of sleep stage transition in whole night sleep. Smoothing rule shows the stable sleep duration and the property of sleep continuity; in other words, in a stable phase of sleep, it is unlikely that the stage suddenly changed. Transition-probability-based rule represents the most probable transition between different stages, which reflects the dynamic property of whole night sleep.

Algorithm 1 shows the pseudo-code of our automatic correction rules construction algorithm, the $T$ and $P$ are computed by GetTransMatAndHighProbPath procedure (shown in Algorithm 2). The subsequences are extracted from prepredicted stage sequence (i.e., the prediction from the random forest classifier) using a sliding window, where the window size is equal to three and five; i.e., each subsequence contains sleep stages for three and five consecutive epochs, such as [Wake, REM, S2] and [Wake, S3, S3, REM, S1]. In line 2-15, the correction rules are applied to extracted subsequences. All the correction rules are described as follows:

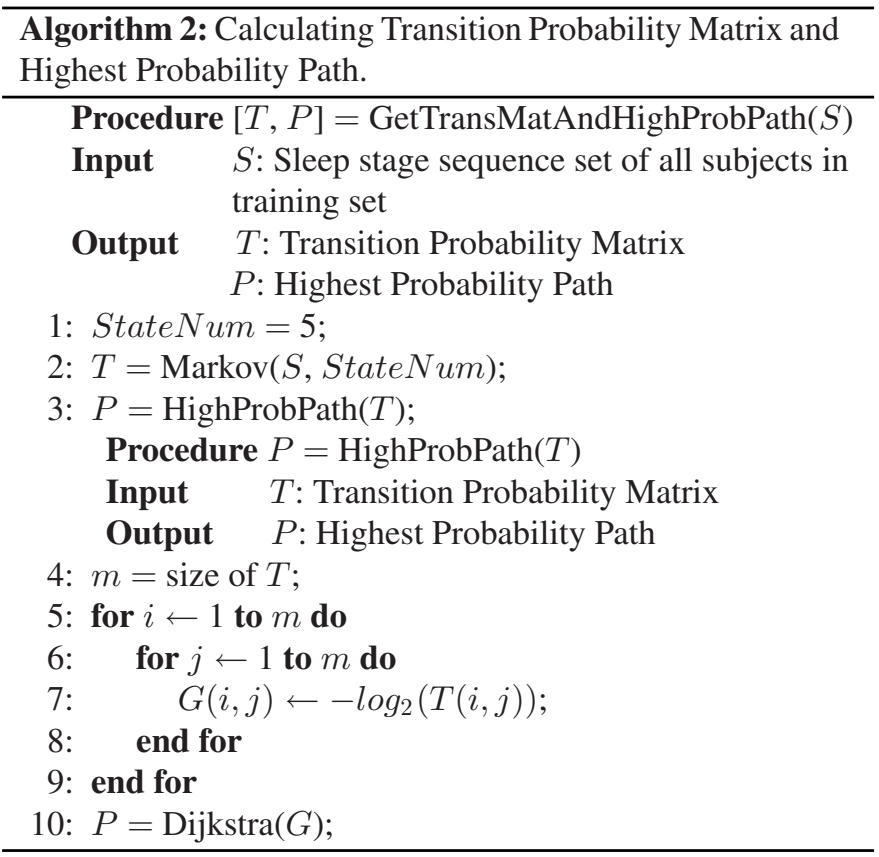

1) Smoothing Rule (Line 7 and 11): Because sleep is a smooth, stable and continuous process [26], it is reasonable to assume that the sleep stage should not change suddenly in 3-epoch subsequence since each epoch only includes 30s of EEG signal. This rule is used to correct the sudden stage change in pre-predicted result. For example, the subsequence [Wake, S3, Wake] is corrected to [Wake, Wake, Wake]. Additionally, according to our observation, this rule can also be extended to 4-epoch subsequence and 5-epoch subsequence; for example, [Wake, S3, S3, Wake, Wake] is corrected to [Wake, Wake, Wake, Wake, Wake]. There is one notable thing that 4-epoch subsequence, such as [Wake, S3, Wake, Wake] or [Wake, Wake, S3, Wake], is already covered by 3 -epoch subsequence. Therefore, the Smoothing rule and its input format is generally summarized as following: 1) $[\mathrm{A}, \mathrm{B}, \mathrm{A}]$ is corrected to $[\mathrm{A}, \mathrm{A}, \mathrm{A}]$, and 2) $[\mathrm{A}, \mathrm{B}, \mathrm{B}, \mathrm{A}$, $\mathrm{A}$ ] is corrected to $[\mathrm{A}, \mathrm{A}, \mathrm{A}, \mathrm{A}, \mathrm{A}]$, where different symbols (like "A" or "B") represent the different categories of sleep stage.

2) Transition-Probability-Based Rule (Line 13): It is necessary to find a reasonable way to change improbable subsequences of pre-predicted sleep stages. For example, the pre-predicted stage subsequence is [Wake, REM, S2]; however, in real life, this sequence happens with quite low probability. In this way, we replace low probability transitions with high probability ones.

Algorithm 2 shows the pseudo-code of learning the stage transition probability matrix and selecting the highest probability transition path between stages using the training set. The number of states is set to be 5 (line 1). Then, in line 2, an asymmetric transition probability matrix $T$ between sleep stages is computed using the Markov model. The element $T(i, j)$ represents the transition probability from stage $i$ to stage $j$. In $T$, the self-transition probability is set to be a very small number $\epsilon$ (i.e., $T(i, i)=\epsilon$ ) since the Smoothing rule solves the self-transition correction. Fig. 3 shows the state-transition diagram of all five sleep stages. 


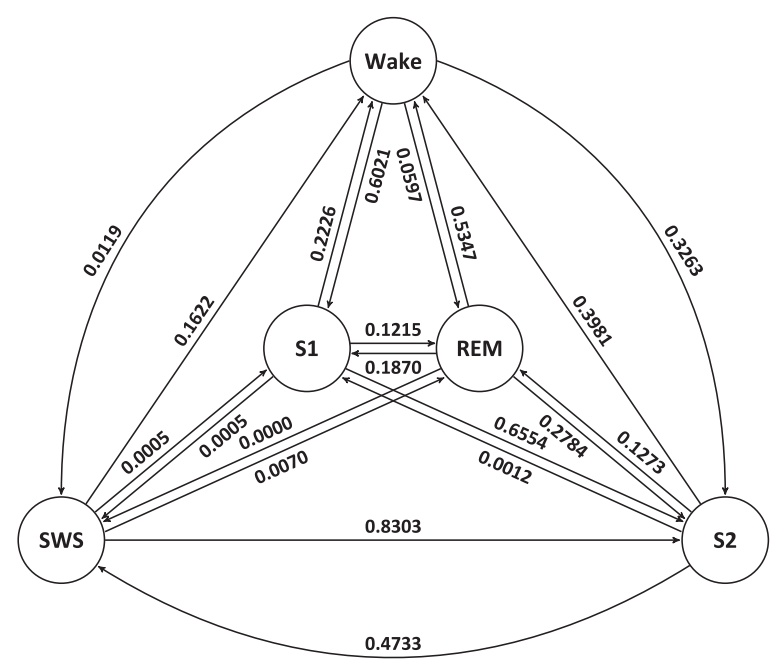

Fig. 3. State-transition diagram of all sleep stages.

Based on the stage transition probability matrix, the highest probability path $P$ among each stage is computed using HighProbPath procedure in line 3, which is shown at the bottom of Algorithm 2. In HighProbPath procedure, the transition probability matrix is transformed to a direct weighted graph $G$ using logarithmic function (line 5-9); each node in $G$ represents the sleep stage, and the edge between node $i$ and node $j$ means the cost of transition. It is easy to prove that the shortest path in $G$ is equal to the highest transition probability path $P$ in $T$. Therefore, in line 10, the Dijkstra algorithm [59] is applied to calculate the shortest path of graph $G$.

Suppose [A, B, C] represents a pre-predicted 3-epoch subsequence as an input of Transition-probability-based rule, where A, B and C are sleep stages of epoch. The corrected sequence (CorrSeq) is generated using (1) shown at the bottom of this page, where $\operatorname{Hpath}(A, B)$ means the highest transition probability path between stage $\mathrm{A}$ and stage $\mathrm{B}$, and $\operatorname{prob}([A, B])$ means the transition probability of the path $[\mathrm{A}, \mathrm{B}]$ according to the transition matrix. Three situations are defined:

1) if the highest transition probability path between stage A and stage $\mathrm{C}$ is $[\mathrm{A}, \mathrm{B}, \mathrm{C}]$, then no rules are applied;

2) if situation 1) is not satisfied and the highest transition probability path between stage $A$ and stage $B$ is $[A, B]$, the find the stage $\mathrm{X}$ with highest transition probability from $\mathrm{B}$ to $\mathrm{X}$, and the corrected sequence is $[\mathrm{A}, \mathrm{B}, \mathrm{X}]$;

3) if both situation 1) and 2) are not satisfied, two paths are computed: $[\mathrm{A}, \mathrm{B}, \mathrm{X}]$ and $[\mathrm{A}, \mathrm{X}, \mathrm{C}]$; then choose the path with highest transition probability as the corrected sequence.
TABLE III

CONFUSION MATRIX

\begin{tabular}{llllll}
\hline \hline & \multicolumn{5}{c}{ Gold Standard } \\
\cline { 2 - 6 } & Wake & REM & $\mathrm{S} 1$ & $\mathrm{~S} 2$ & SWS \\
\hline Predicted & & & & & \\
Wake & $C_{11}$ & $C_{12}$ & $C_{13}$ & $C_{14}$ & $C_{15}$ \\
REM & $C_{21}$ & $C_{22}$ & $C_{23}$ & $C_{24}$ & $C_{25}$ \\
S1 & $C_{31}$ & $C_{32}$ & $C_{33}$ & $C_{34}$ & $C_{35}$ \\
S2 & $C_{41}$ & $C_{42}$ & $C_{43}$ & $C_{44}$ & $C_{45}$ \\
SWS & $C_{51}$ & $C_{52}$ & $C_{53}$ & $C_{54}$ & $C_{55}$ \\
\hline \hline
\end{tabular}

As mentioned before, the pre-predicted stage transition [Wake, REM, S2] is rare in night sleep; Transition-probabilitybased rule generates the stage transition [Wake, S1, S2] with high probability to correct the improbable pre-predicted result; it is more reasonable and consistence with reality [14].

\section{F. Performance Evaluation}

We evaluated the performance of the proposed approach using the recall $R$ (also known as sensitivity), precision $P$ (positive predictive value), F1-measure $(F)$, and accuracy $(A C)$ [30], [31]. For each stage category $c$, its precision $\left(P_{c}\right)$, recall $\left(R_{c}\right)$, and F1-score $\left(F_{c}\right)$ are defined as:

$$
P_{c}=\frac{T P_{c}}{T P_{c}+F P_{c}}, R_{c}=\frac{T P_{c}}{T P_{c}+F N_{c}}, F_{c}=\frac{2 P_{c} \times R_{c}}{P_{c}+R_{c}}
$$

where $T P_{c}$ is the number of true positives, $F P_{c}$ is the number of false positives, and $F N_{c}$ is the number of false negatives. The accuracy $(A C)$ is defined as:

$$
A C=\frac{\text { PredictResults } \cap \text { GroundTruth }}{\text { length }(\text { GroundTruth })}
$$

Additionally, Cohen's kappa coefficient [28], [29] is also an important measure to evaluate the performance of a classifier. It is a more robust measure compared with simple percent agreement calculation. Therefore, we also compute the Cohen's kappa coefficient to evaluate the performance of our approach as described below.

The confusion matrix for classifying 5 sleep stages is shown in Table III, and each row of the matrix represents the instances in a predicted class while each column represents the instances in actual class. The element $C_{i j}$ means the number of common instances between predicted class $i$ and actual class $j$; for example, $C_{12}$ represents the number of instances predicted as Wake, but actually belongs to REM. The kappa coefficient is

$$
\text { CorrSeq }=\left\{\begin{array}{cc}
{[A, B, C]} & \text { if hpath }(A, C)=[A, B, C] \\
{[A, B, X] \text { where } \max _{X \in\{\text { sleep stage }\}}\{\operatorname{prob}([B, X])\}} & \text { elseif hpath }(A, B)=[A, B] \\
\max \left\{\begin{array}{c}
\operatorname{prob}([A, B])+\max _{X \in\{\text { sleep stage }\}}\{\operatorname{prob}([B, X])\} \\
\max _{X \in\{\text { sleep stage }\}}\{\operatorname{prob}([A, Z, X])\}
\end{array}\right\}, & \text { elseif hpath }(A, B) \neq[A, B]
\end{array}\right.
$$




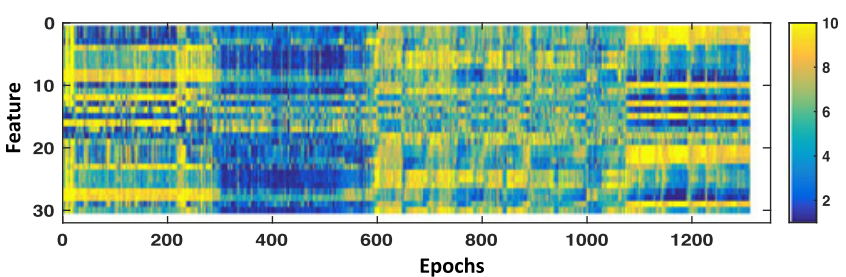

(a)

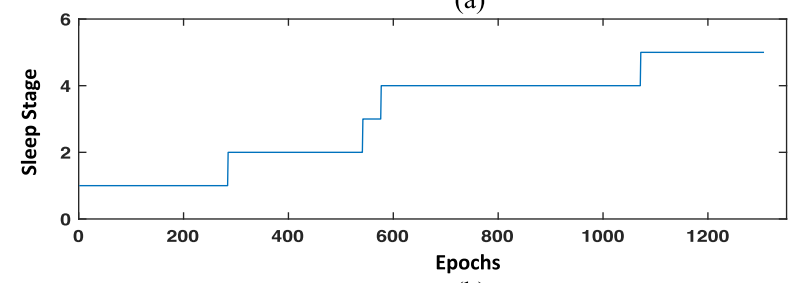

(b)

Fig. 4. Extracted features with corresponding sleep stages. (a) Thirty features of all epochs in a subject's night sleep. The $x$-axis represents indices of epochs, and $y$-axis represents thirty features. Colors represent features' value. (b) Corresponding sleep stages (1: Wake; 2: REM; 3: S1; 4: S2; 5: SWS).

TABLE IV-A

CONFUSION MATRIX OF RANDOM FOREST CLASSIFIER

\begin{tabular}{lccccc}
\hline \hline & Wake & REM & S1 & S2 & SWS \\
\hline Wake & 38505 & 2073 & 1257 & 2907 & 312 \\
REM & 1360 & 14587 & 1772 & 1977 & 1 \\
S1 & 23 & 60 & 67 & 13 & 0 \\
S2 & 1278 & 1654 & 779 & 41121 & 2701 \\
SWS & 54 & 12 & 2 & 2948 & 14583 \\
\hline \hline
\end{tabular}

defined as follows:

$$
\begin{gathered}
K_{a}=\frac{P_{o}-P_{c}}{1-P_{c}}, P_{o}=\frac{\sum_{i=1}^{5} C_{i i}}{\sum_{i=1}^{5} \sum_{j=1}^{5} C_{i j}} \\
P_{c}=\frac{\sum_{i=1}^{5}\left(\sum_{j=1}^{5} C_{i j} \sum_{j=1}^{5} C_{j i}\right)}{\left(\sum_{i=1}^{5} \sum_{j=1}^{5} C_{i j}\right)^{2}}
\end{gathered}
$$

where $P_{o}$ is the proportion of the observed agreements, and $P_{c}$ is the proportion of agreements expected by chance [15]. The interpretation of kappa coefficient has six levels of agreement: 1) poor (less than 0$), 2)$ slight $(0.00$ to 0.20$), 3)$ fair (0.21 to $0.40), 4)$ moderate $(0.41$ to 0.60$), 5)$ substantial $(0.61$ to 0.80$)$, and 6$)$ excellent $(>0.8)[14]$.

\section{EXPERIMENTS AND RESULTS}

\section{A. Classification Results}

We processed, filtered, and segmented the EEG signal into 30s epochs and extracted thirty-three features int time domain, frequency domain and non-linear fields. According to our analysis, the mean, mobility and complexity were not used for classification, since they had similar value for each stage. Fig. 4 shows the extracted 30 features' value with their corresponding sleep stage for one subject with 10-bins; different sleep stages have distinct feature patterns.
TABLE IV-B

Performance of Random Forest Classifier

\begin{tabular}{lccccr}
\hline \hline & Wake & REM & S1 & S2 & SWS \\
\hline Recall (\%) & 93.41 & 79.33 & 01.73 & 83.98 & 82.87 \\
Precision (\%) & 85.46 & 74.05 & 41.10 & 86.51 & 82.86 \\
F1-score (\%) & 89.26 & 76.60 & 03.31 & 85.22 & 82.86 \\
\multicolumn{5}{c}{ Accuracy: 83.59\% Kappa: 0.7715 } \\
\hline \hline
\end{tabular}

TABLE V-A

CONFUSION MATRIX OF HYCLASSS

\begin{tabular}{lccccc}
\hline \hline & Wake & REM & S1 & S2 & SWS \\
\hline Wake & 38450 & 1226 & 1300 & 1875 & 153 \\
REM & 985 & 15751 & 1454 & 1627 & 0 \\
S1 & 109 & 70 & 284 & 174 & 4 \\
S2 & 1587 & 1337 & 839 & 42925 & 2901 \\
SWS & 89 & 2 & 0 & 2365 & 14539 \\
\hline \hline
\end{tabular}

TABLE V-B

Performance of HyClASsS

\begin{tabular}{lccccr}
\hline \hline & Wake & REM & S1 & S2 & SWS \\
\hline Recall (\%) & 93.27 & 85.67 & 07.33 & 87.66 & 82.62 \\
Precision (\%) & 89.41 & 79.48 & 44.31 & 86.56 & 85.55 \\
F1-score (\%) & 91.30 & 82.46 & 12.57 & 87.11 & 84.60 \\
\hline \multicolumn{7}{c}{ Accuracy: 85.95\% Kappa: 0.8046 } \\
\hline
\end{tabular}

We randomly assigned subjects in the CCSHS and CFS datasets to either a training set or a testing set. The resulting training set included 96 subjects; the testing set contained the remaining 102 subjects. We compared the output of the classifier with the gold standard to evaluate its performance. In the experiment, the number of $\mathrm{N}$-bin and trees in random forest were set to 100 and 400, respectively; Section IV-B discusses the selection of these two parameters.

Table IV-A and IV-B show the evaluation results for random forest classifier without correction rules applied, including the confusion matrix, F1-score, recall and precision of each stage class, overall accuracy and kappa coefficient. The overall average accuracy was $83.59 \%$ for each stage class of all subjects. Except for S1, the average recall was higher than $79 \%$, the average precision was higher than $74 \%$, and the average F1-score was higher than $76 \%$. It also indicated that the average kappa was in substantial agreement level (0.7715). In contrast, Table V-A and V-B show the evaluated results for random forest classifier with correction rules applied, i.e., the HyCLASSS. The overall accuracy increased to $85.95 \%$; the average recall, average precision and average F1-score for each stage class improved significantly, especially for REM and S1. As mentioned before, the signal features of REM and S1 are similar; in addition, the number of S1's epochs was significantly smaller than other stages' epochs in a subject's night sleep. This makes it difficult to train a model with a high performance for S1 as S1 could be easily mistakenly classified as one of the other stages. After using correction rules, the F1-score results for REM and 


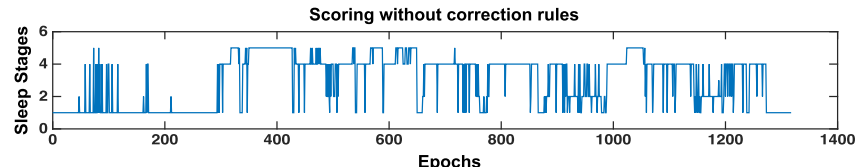

(a)

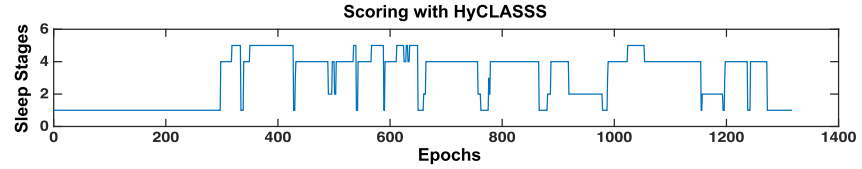

(b)

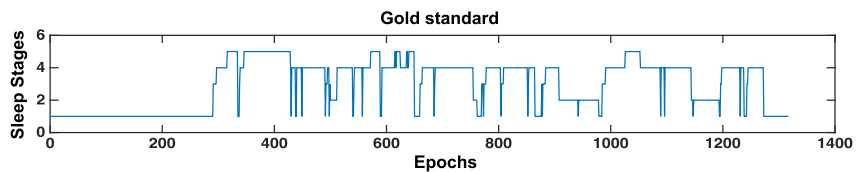

(c)

Fig. 5. The sleep stage sequences of a subject in testing set: (a) the automatic scoring without correction rules, (b) the automatic scoring with HyCLASSS, and (c) gold standard. The x-axis represents the indices of epochs, and y-axis represents 5 sleep stages (1: Wake; 2: REM; 3: S1; 4: S2; 5: SWS).

S1 were both increased by $5.86 \%$ (from $76.60 \%$ to $82.46 \%$ ) and $9.26 \%$ (from $3.31 \%$ to $12.57 \%$ ), respectively. Moreover, the average kappa was increased from 0.7715 to 0.8046 , which was in excellent agreement level $(>0.8)$.

Fig. 5 shows the stage sequences of one subject (No. 15 in testing set) including the gold standard manually scored by the expert, the automatic stage scoring without correction rules applied, and the automatic scoring with HyCLASSS. The resulting stage sequence scored with HyCLASSS was much closer to the gold standard after the rule correction.

\section{B. Parameter Selection}

Parameter selection is an important problem in classification as changing parameters may impact significantly the performance of the classifier. As mentioned before, there were two parameters in HyCLASSS: the number of N-bin for feature ranking and the number of trees in random forest. In order to verify the sensitivity, we tested the performance using various parameters ranges: 1) the number of trees was set to 400 and the number of N-bin ranged form 10 to 2000;2) the number of $\mathrm{N}$-bin was set to 100 and the number of trees ranged form 10 to 1000. Fig. 6 shows the results of experiments. The average accuracy and kappa coefficient for different parameters only had slightly changes. It indicated that HyCLASSS is not sensitive to its parameters.

\section{DISCUSSION}

In this study, we proposed an automatic sleep stage classification and prediction approach based on the hybrid classifier HyCLASSS. It combines a random forest classifier and correction rules learned from Markov model. The main idea of HyCLASSS is to leverage both the advantages of signal features and the sleep stage dynamic transition feature.

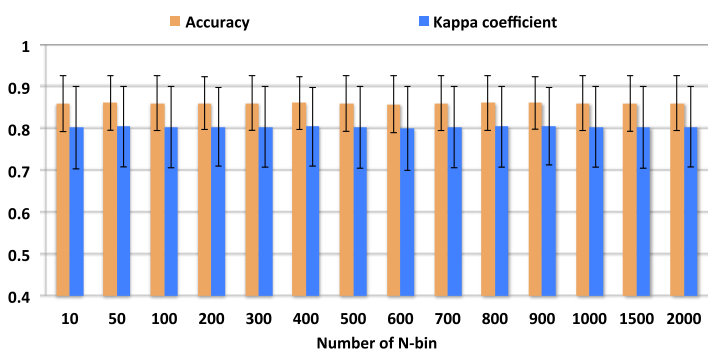

(a)

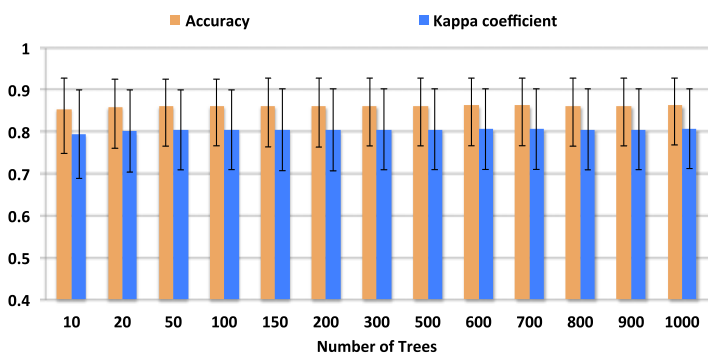

(b)

Fig. 6. The average accuracy and kappa coefficient for different parameters, (a) number of $\mathrm{N}$-bin and (b) number of trees in random forest.

TABLE VI

The Average Kolmogorov Complexity of Stage Sequences AND RANDOM SEQUENCES (198 SUBJECTS)

\begin{tabular}{lc}
\hline Stage Sequences & Random Sequences \\
\hline $0.4203( \pm 0.081)$ & $2.1972( \pm 0.025)$ \\
\hline \hline
\end{tabular}

In Section III-E, we designed an experiment to compare the sleep stage sequence with random sequence using Kolmogorov complexity. The process of this experiment is summarized as follows: for each subject, 1) calculate the Kolmogorov complexity of stage sequence $K_{s} ; 2$ ) generate a random sequence that has the same length with the stage sequence and calculate its Kolmogorov complexity value $K_{r}$. The results are shown in Table VI. Higher value of Kolmogorov complexity indicates higher randomness. The results indicate that the stage sequence of whole night sleep is not a random sequence. Therefore, learning stage transition rules can provide more information and knowledge about human sleep, and we showed that it improved the performance of classifiers.

In previous studies, several approaches have been applied to automated sleep stage scoring. Hae-Jeong et al. [12] used a rule-based and artificial neural networks approach based on 58 features, which they extracted from EEG, EOG and EMG signals, to perform automatic sleep scoring. It had a performance of $85.9 \%$ for 4 subjects. Masaaki et al. [21] proposed a method using $\mathrm{C} 4.5$ decision tree and applied it to 6 features extracted from EEG, EOG and EMG signals. Their accuracy was $81.4 \%$ for 5 subjects. In 2009, a model based on Hilbert-Huang transform, proposed by Li et al. [16], showed an average accuracy of $81.70 \%$ in 16 sleep records from a single EEG channel. In the same year, Fraiwan et al. [17] proposed a classifier of regression trees based on 54 extracted wavelet features from EEG, and re- 
TABLE VII

COMPARISON WITH PREVIOUS WORK

\begin{tabular}{|c|c|c|c|c|c|c|c|}
\hline Author & Year & Signal Type & Stage No. & Method & Feature No. & Dataset Size & $\operatorname{Acc}(\%)$ \\
\hline HaeJeong Park [12] & 2000 & $\mathrm{EEG}+\mathrm{EOG}+\mathrm{EMG}$ & 6 & Rule-based + ANN & 58 & 4 subjects & 85.90 \\
\hline Masaaki Hanaoka [21] & 2001 & $\mathrm{EEG}+\mathrm{EOG}+\mathrm{EMG}$ & 7 & C4.5 decision tree & 6 & 5 subjects & 81.40 \\
\hline Luay A. Fraiwan [17] & 2009 & EEG & 6 & Wavelet + Regression tree & 20 & 936 epochs & 74.00 \\
\hline Yi Li [16] & 2009 & EEG & 4 & Hilbert-Huang transform $+\mathrm{KNN}$ & 6 & 16 subjects & 81.70 \\
\hline Han G. Jo [4] & 2010 & $\mathrm{EEG}+\mathrm{EOG}+\mathrm{EMG}$ & 4 & Fuzzy classifier & 5 & 4 subjects & 84.60 \\
\hline Sheng-Fu Liang [14] & 2012 & $\mathrm{EEG}+\mathrm{EOG}+\mathrm{EMG}$ & 5 & Rule-based decision tree & 12 & 17 subjects & 86.70 \\
\hline B. Koley [7] & 2012 & EEG & 5 & SVM feature selection + SVM & 21 & 28 subjects & 85.70 \\
\hline Luay Fraiwan [15] & 2012 & EEG & 5 & Wavelet + Random forest & 7 & 16 subjects & 83.00 \\
\hline Martin Lańgkvist [13] & 2012 & $\mathrm{EEG}+\mathrm{EOG}+\mathrm{EMG}$ & 5 & Deep Belief Networks & 0 & 25 subjects & 64.70 \\
\hline Pan, S.-T. [25] & 2012 & $\mathrm{EEG}+\mathrm{EOG}+\mathrm{EMG}$ & 5 & Discrete Hidden Markov Model & 13 & 20 subjects & 85.30 \\
\hline Jose Luis Rodrguez-Sotelo [20] & 2014 & EEG & 5 & EEG entropy measures + Unsupervised classification & 13 & 22 subjects & 80.00 \\
\hline Our Approach & & EEG & 5 & HyCLASSS & 30 & 198 subjects & 85.95 \\
\hline
\end{tabular}

ported a 74\% accuracy for all 936 epochs. Han G. Jo et al. [4] proposed a fuzzy classifier based on 5 features extracted from EEG, EOG and EMG signals that had an accuracy of $84.6 \%$ for 5 subjects. Sheng Fu et al. [14] proposed a rule-based decision tree approach in 2012 based on 12 EEG, EOG and EMG signal features which showed an accuracy of $86.7 \%$ with 17 subjects. In the same year, Koley et al. [7] built a model based on SVM feature selection and SVM classifier. 39 features were extracted from EEG signals, and the model was applied to classifying 28 subjects' sleep records; their final accuracy was $85.7 \%$. Fraiwan et al. [15] proposed a method using random forest based on 7 EEG signal wavelet features, and their average accuracy and kappa coefficient were $83 \%$ and 0.76 for 16 subjects. Längkvist et al. [13] proposed a method with Deep Belief Network based on original EEG signals (no extracted features), and their average accuracy was $67.4 \%$ for 25 subjects. Pan et al. [25] proposed a method using Discrete Hidden Markov Model and applied to 13 features extracted from EEG, EOG and EMG signals; their accuracy was $85.3 \%$ for 20 subjects. Rodrguez-Sotelo et al. [20] built a model based on unsupervised feature classification algorithms with 13 EEG entropy measures, the model was applied to classify 22 subjects' sleep records and had an accuracy of $80 \%$. All the studies mentioned above are summarized in Table VII.

In our experiment, the random forest classifier turned out to be a useful tool for classifying sleep stages with an accuracy of $83.59 \%$, and a kappa coefficient of 0.7715 , which indicates a substantial agreement. A group of correction rules, learned from Markov model, was applied to the classification output of this random forest. This improved overall agreement of the HyCLASSS to $85.95 \%$. Additionally, the average Cohen's kappa coefficient for 102 testing subjects was 0.8046 and represents an excellent agreement with the gold standard. Compared with previous works, HyCLASSS was applied to a much larger dataset, which included 198 subjects, and our results indicate that the performance of HyCLASS compared favorably to previous work for using a single EEG channel. Additionally, another advantage of HyCLASSS is that it is insensitive to the changing of parameters.

For computational complexity, the overall cost of feature extraction is $O\left(n^{2}\right)$, where $n$ is the number of data points in an EEG recording. This is because the non-linear dynamics features such as largest Lyapunov exponent and Kolmogorov entropy have complexity of $O\left(n^{2}\right)$ [60], [61]. When using the random forest classifier method, building a single tree has time complexity $O(\operatorname{mnlog}(n))$, where $n$ is the number of instances and $m$ is the number of attributes. The total time complexity for building a random forest is therefore $O(\operatorname{pmn} \log (n))$ with $p$ trees [62]. Our experiments were performed on a computer with Intel Core i7 (3.1 GHz) CPU and $16 \mathrm{~GB}$ memory. For feature extraction, our average computational time was 4,374 seconds per subject. For classification, the training and testing time for all subjects were 779 and 795 seconds, respectively.

Another significance of our approach is that, to the best of our knowledge, this is the first time both signal and stage transition features of sleep have been applied to automatic sleep stage scoring. HyCLASSS provides a new aspect on scoring sleep data. These two kinds of features reflect the different characteristics of sleep; one represents the signal changes of each stage, for example, SWS has more lower frequency components. The other stage transition features show the stage dynamic changes during the night sleep. For example, it is improbable to transition from REM to SWS directly, and there has to be one or more transition stages between them, like S2. The major difference between whole night sleep stage scoring and the regular classification problem is that the label sequence of sleep stages is another valuable source of information, since the stage sequence is not a random sequence, and there are underline relationships between stages. These relationships are useful for discovering new mechanisms of sleep regulation and its evolution, and it may provide help in predicting specific outcomes [27].

Our study has some limitations: 1) only signals from a single EEG channel were used in classification, 2) the correction rules may not be suitable for all situations, 3 ) we have not considered the effects of diseases, such as narcolepsy, insomnia and sleep disorder, on their influences on classification. In future work, we will focus on sleep stage scoring using multiple PSG signals, such as EOG and EMG, to improve the performance of our approach. Moreover, we will design more elegant algorithms for analyzing and classifying sleep data. Additionally, we will apply our approach to more datasets, especially to the datasets 
of different sleep diseases studies, which can be obtained from the NSRR website [9].

\section{CONCLUSION}

A hybrid classifier (HyCLASSS), combining the benefits of random forest classifier and correction rules has been proposed for automatic sleep stage scoring with single channel EEG recordings. Several features from temporal, frequency and nonlinear analyses provided valuable information to characterize sleep stages. Random forest classifiers were shown to be a useful tool to obtain high accuracy sleep stage classification from EEG recordings. The automatically constructed correction rules, which were based on the dynamic stage transition feature, significantly improved the performance of the classifier. The results indicated an excellent agreement between automatic scoring and the gold standard.

\section{REFERENCES}

[1] S. Panda, J. B. Hogenesch, and S. A. Kay, "Circadian rhythms from flies to human," Nature, vol. 417, no. 6886, pp. 329-335, 2002.

[2] M.-H. Chung, T. B. Kuo, N. Hsu, H. Chu, K.-R. Chou, and C. C. Yang, "Sleep and autonomic nervous system changes? Enhanced cardiac sympathetic modulations during sleep in permanent night shift nurses," Scand. J. Work, Environ. Health, vol. 35, pp. 180-187, 2009.

[3] J. M. Ellenbogen, J. D. Payne, and R. Stickgold, "The role of sleep in declarative memory consolidation: Passive, permissive, active or none?" Current Opinion Neurobiol., vol. 16, no. 6, pp. 716-722, 2006.

[4] H. G. Jo, J. Y. Park, C. K. Lee, S. K. An, and S. K. Yoo, "Genetic fuzzy classifier for sleep stage identification," Comput. Biol. Med., vol. 40, no. 7 , pp. 629-634, 2010.

[5] E. A. Wolpert, "A manual of standardized terminology, techniques and scoring system for sleep stages of human subjects," Arch. Gen. Psychiatry, vol. 20, no. 2, 1969, Art. no. 246.

[6] V. Jääskinen, "Modelling sleep stages with Markov chains," 2009.

[7] B. Koley and D. Dey, "An ensemble system for automatic sleep stage classification using single channel EEG signal," Comput. Biol. Med., vol. 42 , no. 12, pp. 1186-1195, 2012.

[8] D. Dean et al., "Scaling up scientific discovery in sleep medicine: The national sleep research resource," Sleep, vol. 39, no. 5, pp. 1151-64, 2016.

[9] National sleep research resource website. [Online]. Available: https://sleepdata.org/

[10] R. Maulsby, J. Frost, and M. Graham, "A simple electronic method for graphing EEG sleep patterns," Electroencephalogr. Clin. Neurophysiol., vol. 21, no. 5, pp. 501-503, 1966.

[11] H. W. Agnew, J. C. Parker, W. B. Webb, and R. L. Williams, "Amplitude measurement of the sleep electroencephalogram," Electroencephalogr. Clin. Neurophysiol., vol. 22, no. 1, pp. 84-86, 1967.

[12] H. Park, K. Park, and D.-U. Jeong, "Hybrid neural-network and rule-based expert system for automatic sleep stage scoring," in Proc. 22nd Annи. Int. Conf. IEEE Eng. Med. Biol. Soc., 2000, vol. 2, pp. 1316-1319.

[13] M. Längkvist, L. Karlsson, and A. Loutfi, "Sleep stage classification using unsupervised feature learning," Adv. Artif. Neural Syst., vol. 2012, 2012, Art. no. 5 .

[14] S.-F. Liang, C.-E. Kuo, Y.-H. Hu, and Y.-S. Cheng, "A rule-based automatic sleep staging method," J. Neurosci. Methods, vol. 205, no. 1, pp. 169$176,2012$.

[15] L. Fraiwan, K. Lweesy, N. Khasawneh, H. Wenz, and H. Dickhaus, "Automated sleep stage identification system based on time-frequency analysis of a single EEG channel and random forest classifier," Comput. Methods Programs Biomed., vol. 108, no. 1, pp. 10-19, 2012.

[16] Y. Li, F. Yingle, L. Gu, and T. Qinye, "Sleep stage classification based on EEG hilbert-huang transform," in Proc. 4th IEEE Conf. Ind. Electron. Appli., 2009, pp. 3676-3681.

[17] L. Fraiwan, N. Khaswaneh, and K. Lweesy, "Automatic sleep stage scoring with wavelet packets based on single EEG recording," in Proc. World Acad. Sci., Eng. Technol, Paris, France, 2009, vol. 54, pp. 385-488.

[18] F. Ebrahimi, M. Mikaeili, E. Estrada, and H. Nazeran, "Automatic sleep stage classification based on EEG signals by using neural networks and wavelet packet coefficients," in Proc. 30th Annu. Int. Conf. IEEE Eng. Med. Biol. Soc., 2008, pp. 1151-1154.

[19] C.-F. V. Latchoumane and J. Jeong, "Quantification of brain macrostates using dynamical nonstationarity of physiological time series," IEEE Trans. Biomed. Eng., vol. 58, no. 4, pp. 1084-1093, Apr. 2011.

[20] J. L. Rodríguez-Sotelo, A. Osorio-Forero, A. Jiménez-Rodríguez, D. Cuesta-Frau, E. Cirugeda-Roldán, and D. Peluffo, "Automatic sleep stages classification using eeg entropy features and unsupervised pattern analysis techniques," Entropy, vol. 16, no. 12, pp. 6573-6589, 2014.

[21] M. Hanaoka, M. K. Ashi, and H. Yamazaki, "Automated sleep stage scoring by decision tree learning," in Proc. 23rd Annu. Int. Conf. IEEE Eng. Med. Biol. Soc., 2001, vol. 2, pp. 1751-1754.

[22] S. Güneş, K. Polat, and Ş. Yosunkaya, "Efficient sleep stage recognition system based on EEG signal using k-means clustering based feature weighting," Expert Syst. Appl., vol. 37, no. 12, pp. 7922-7928, 2010.

[23] D. Novák, Y. Al-Ani, and L. Lhotska, "Electroencephalogram processing using hidden Markov models," in Proc. 5th EUROSIM Congr. Model. Simul., 2004

[24] L. Doroshenkov and V. Konyshev, "Usage of hidden Markov models for automatic sleep stages classification," in Proc. Russ.-Bavarian Conf. Biomed. Eng., 2007, Paper 19.

[25] S.-T. Pan, C.-E. Kuo, J.-H. Zeng and, S.-F. Liang, "A transitionconstrained discrete hidden Markov model for automatic sleep staging," Biomed. Eng. Online, vol. 11, 2012, Art no. 52.

[26] A. Laffan, B. Caffo, B. J. Swihart, and N. M. Punjabi, "Utility of sleep stage transitions in assessing sleep continuity," Sleep, vol. 33, no. 12, 2010, Art no. 1681

[27] A. Kishi, Z. R. Struzik, B. H. Natelson, F. Togo, and Y. Yamamoto, "Dynamics of sleep stage transitions in healthy humans and patients with chronic fatigue syndrome," Amer. J. Physiol-Regulatory, Integrative Comparative Physiol., vol. 294, no. 6, pp. R1980-R1987, 2008.

[28] J. R. Landis and G. G. Koch, "The measurement of observer agreement for categorical data," Biometrics, vol. 33, no. 1, pp. 159-174, 1977.

[29] J. Sim and C. C. Wright, "The kappa statistic in reliability studies: Use, interpretation, and sample size requirements," Phys. Therapy, vol. 85, no. 3 , pp. 257-268, 2005.

[30] C. J. van Rijsbergen, Information Retrieval. London, U.K.: Butterworth, 1979 .

[31] D. M. W. Powers, "Evaluation: From precision, recall and f-measure to ROC, informedness, markedness and correlation," J. Mach. Learn. Technol., vol. 2, no. 1, pp. 37-63, 2011.

[32] T.-C. Fu, "A review on time series data mining," Eng. Appl. Artif. Intell., vol. 24 , no. 1 , pp. 164-181, 2011

[33] N. V. Thakor and S. Tong, "Advances in quantitative electroencephalogram analysis methods," Аnnu. Rev. Biomed. Eng., vol. 6, pp. 453-495, 2004.

[34] B. Hjorth, "EEG analysis based on time domain properties," Electroencephalogr. Clin. Neurophysiol., vol. 29, no. 3, pp. 306-310, 1970.

[35] J. Jobson, Applied Multivariate Data Analysis: Volume II: Categorical and Multivariate Methods. Berlin, Germany: Springer, 2012.

[36] S. Charbonnier, L. Zoubek, S. Lesecq, and F. Chapotot, "Self-evaluated automatic classifier as a decision-support tool for sleep/wake staging," Comput. Biol. Med., vol. 41, no. 6, pp. 380-389, 2011.

[37] S. J. Redmond and C. Heneghan, "Cardiorespiratory-based sleep staging in subjects with obstructive sleep apnea," IEEE Trans. Biomed. Eng., vol. 53, no. 3, pp. 485-496, Mar. 2006.

[38] D. Hord, L. Johnson, A. Lubin, and M. Austin, "Resolution and stability in the autospectra of EEG," Electroencephalogr. Clin. Neurophysiol., vol. 19, no. 3, pp. 305-308, 1965.

[39] J. Fell, J. Röschke, K. Mann, and C. Schäffner, "Discrimination of sleep stages: A comparison between spectral and nonlinear EEG measures," Electroencephalogr. Clin. Neurophysiol., vol. 98, no. 5, pp. 401-410, 1996.

[40] R. Acharya, O. Faust, N. Kannathal, T. Chua, and S. Laxminarayan, "Nonlinear analysis of EEG signals at various sleep stages," Comput. Methods Programs Biomed., vol. 80, no. 1, pp. 37-45, 2005.

[41] L. Breiman, "Random forests," Mach. Learn., vol. 45, no. 1, pp. 5-32, 2001

[42] Y. H. Hu and J.-N. Hwang, Handbook of Neural Network Signal Processing. Boca Raton, FL, USA: CRC Press, 2001.

[43] T. Joachims, "Making large scale SVM learning practical," in Advances in Kernel Methods-Support Vector Learning, B. Schölkopf, C. J. C. Burges, and A. J. Smola, Eds. Cambridge, MA: MIT Press, 1998.

[44] S. R. Safavian and D. Landgrebe, "A survey of decision tree classifier methodology," IEEE Trans. Syst. Man, Cybern., vol. 21, no. 3, pp. 660-674, May/Jun. 1991. 
LI et al.: HYCLASSS: A HYBRID CLASSIfiER FOR AUTOMATIC SLEEP STAGE SCORING

385

[45] Cleveland children's sleep and health study. National Sleep Research Resource, Boston, MA, USA. [Online]. Available: https://sleepdata. org/datasets/ccshs

[46] The cleveland family study. National Sleep Research Resource, Boston, MA, USA. [Online]. Available: https://sleepdata.org/datasets/cfs

[47] N. Brian, Y. Gao, B. Wang, F. Gu, and L. Chang, "Prediction of epileptic seizures based on second-order c_(0) complexity," OALib Journal, 2007.

[48] F. Chen, F. Gu, J. Xu, Z. Lu, and R. Lieu, "A new measurement of complexity for studying eeg mutual information," Shengwu Wuli Xuebao, vol. 14, no. 3, pp. 508-512, 1997.

[49] J. Bruhn, L. E. Lehmann, H. Röpcke, T. W. Bouillon, and A. Hoeft, "Shannon entropy applied to the measurement of the electroencephalographic effects of desflurane," J. Amer. Soc. Anesthesiologists, vol. 95, no. 1 , pp. 30-35, 2001.

[50] C. E. Shannon, "A mathematical theory of communication," ACM SIGMOBILE Mobile Comput. Commune. Rev., vol. 5, no. 1, pp. 3-55, 2001.

[51] J. Fell, J. Röschke, and P. Beckmann, "Deterministic chaos and the first positive lyapunov exponent: A nonlinear analysis of the human electroencephalogram during sleep," Biol. Cybern., vol. 69, no. 2, pp. 139-146, 1993.

[52] I. Rezek and S. J. Roberts, "Stochastic complexity measures for physbiological signal analysis," IEEE Trans. Biomed. Eng., vol. 45, no. 9 , pp. 1186-1191, Sep. 1998.

[53] T. Inouye et al., "Quantification of EEG irregularity by use of the entropy of the power spectrum," Electroencephalogr. Chin. Neurophysiol., vol. 79, no. 3, pp. 204-210, 1991.
[54] H. Kantz and T. Schreiber, Nonlinear Time Series Analysis, vol. 7. Cambridge, U.K.: Cambridge Univ. Press, 2004.

[55] M. Li and P. Vitányi, An Introduction to Kolmogorov Complexity and Its Applications. Berlin, Germany: Springer, 2013.

[56] P. Grunwald and P. Vitányi, "Shannon information and Kolmogorov complexity," IEEE Trans. Information Theory, Sep. 2004.

[57] L. Fortnow, "Kolmogorov complexity," in Aspects of Complexity, vol. 4. New Zealand: New Zealand Mathematical Research Intitote (Short Courses in Complexity from the New Zealand Mathematical Research Institute Summer 2000 Meeting, Kaikoura), 2001, pp. 73-86.

[58] R. B. Berry, R. Brooks, C. E. Gamaldo, S. Harding, C. Marcus, and B. Vaughn, The AASM manual for the scoring of sleep and associated events Rules, Terminology and Technical Specifications. Darien, IL, USA: American Academy of Sleep Medicine, 2012.

[59] E. W. Dijkstra, "A note on two problems in connexion with graphs," Numen. Math., vol. 1, no. 1, pp. 269-271, 1959.

[60] M. T. Rosenstein, J. J. Collins, and C. J. De Lucan , "A practical method for calculating largest Lyapunov exponents from small data sets," Phys. D, Nonlinear Phenom., vol. 65, no. 1/2, pp. 117-134, 1993.

[61] A. Teixeira, A. Maros, A. Souto, and L. Antunes, "Entropy measures vs. Kolmogorov complexity," Entropy, vol. 13, no. 3, pp. 595-611, 2011.

[62] G. Beau, "Analysis of a random forests model," J. Mach. Learn. Res., vol. 13, no. 1, pp. 1063-1095, 2012. 